\title{
HOW TO SOLVE AN OPERATOR EQUATION
}

\author{
MARTIN MATLILEU
}

\begin{abstract}
This article summarizes in series of lectumes delivered at the Mathematics Department of the Liniversity of Lejpzig, Cormany in A pril 199l, which were to overview lechnicues for solving operator equations on $C^{*}$-algebras connected with methods developed in a Spanish-Germats rescarch project on "Structure and Applications of $C^{*}$-Algebrats of Quotients" (SACQ). One of the rescarchers in this project was Professor Pcre Mettal until lis unexpected death this A pril. To his memory this paper shall be dedicated.
\end{abstract}

\section{Introduction}

Solving equations belongs to the fundamental tasks of mathenatics. Many problems in the sciences lead to equations involving numbers, mappings, and other quantities. In fact, it frequently occurs that eventually a question can be phresed as an "equation", although, at first; it appeared to be of a rather different nature. To find a solution of an equation generally inplies both the existence as well as the unicueness problem. There is no universal procedure for solving; but the devices invented secn to be as manifold as the possible questions, and only allow a rather rough classification such as numerical, approximative, algebraic methods ctc. However, it is always an importanti step to determine the common fedtures in solving a certain class of examples for the ain of developing a machinery which enables to handle a specified collection of equations at. one time.

In the present paper; we will be concerned with equations within a nor-commutative infinite dimensional setting. To be more specific, they will be of the form

$$
T_{c y, z_{1}, \ldots, x_{n}}=0
$$

\footnotetext{
This paper is part of a researcls project supported by the DAAD.
} 
where, for each 'parameter' $\alpha, T_{\alpha, x_{1}, \ldots, x_{n}}$ is a linear operator on a $C^{*}$ algebra $A$ (with certain additional properties) and we are looking for elements $x_{j} \in A$ solving the equation (1) (or better, this system of equations). We will firstly collect some examples of questions which can be phrased in an equation such as (1), then describe a general tool to tackle them, and finally indicate solutions which yield answers to the questions listed. As a cornmon feature, the questions in Section 2 lead to equations in a $C^{*}$-algebra, that is, we are looking for certain elements in a $C^{*}$-algebra solving the equation, while the conditions typically are formulated in terms of operators defined on the $C^{*}$-algebra. Needless to say that there are many more instances which can be settled by the proposed methods.

\section{Examples}

We have selected our examples from the following four classes of operators on $C^{*}$-algebras: derivations, completely positive operators, centralizing mappings, and generators of dynamical semigroups.

\subsection{Derivations.}

Let $A$ be a $C^{*}$-algebra and $\delta$ a derivation on $A$, i.e. a linear mapping from $A$ into itself satisfying Leibniz' rule $\delta(x y)=x \delta(y)+\delta(x) y$ for all $x, y \in A$. Each derivation $\delta$ is automatically bounded whence it is meaningful and worthwhile to know under which circurnstances $\delta$ is a compaci operator, with respect to the norm or a weaker topology. Here, we ask when $\delta$ is weakly compact, that is, when does $\delta$ map the unit ball of $A$ into a subset whose closure is compact with respect to the weak topology on $A$. (This is more closely related to the point of view taken in this paper than the norm compact case, which, however, can be treatcd sinilarly.)

Specialize to the case $A=B(H)$, the algebra of all bounded linear operators on some Hilbert space $H$. Since $B(H)$ is the second dual of $K(H)$, the closed ideal of all compact, operators on $H$, and $\delta$ is continuous with respect to the $\sigma\left(B(H), K(H)^{*}\right)$-topology, $\delta$ coincides with $\left(\delta_{1}\right)^{* *}$, the second adjoint of the restriction $\delta_{1}$ of $\delta$ to $K(H)$. It is well known that $\delta_{1}$ is weakly compact if and only if $\left(\delta_{1}\right)^{* *}$ maps $K(H)^{* *}$ into $K(H)\{\mathbf{1 5}$, VI.4.2]. Moreover, by Gantmacher's theorem [15, VI.4.8], $\delta$ is weakly compact if and only if $\left(\delta_{1}\right)^{* *}$ is weakly compact. Putting all this together yields that $\delta=\left(\delta_{1}\right)^{* *}$ is weakly compact if and only if $\delta B(H) \subseteq K(H)$.

In the general case we have to replace $K(H)$ by the ideal $K(A)$ of all compact elements in $A$, and, using appropriate representations, we obtain the following, of. [23, Theorern 2.7]. 
Proposition 1. A derivation $\delta$ on a $C^{*}$-algebra $A$ is weakly compact if and only if $\delta^{* *} A^{* *} \subseteq K(A)$.

Again, $K(A)$ is $\delta$-invariant and thus $\delta$ induces a derivation $\bar{\delta}$ on the generalized Calkin algebra $A / K(A)$.

Corollary 2. If $\delta$ is weakly compact, then $\tilde{\delta}=0$.

Suppose $\delta$ were inner, i.e. $\delta=\delta_{a n}$, where $\delta_{a}(x)=x a-a x$, and the element $a$ belonged to $K(A)$. Then, $\delta$ is weakly compact by $[\mathbf{4 1}$, Theorem 3.1]. On the other hand, $\delta^{* *}$ is always inner by Sakai's theorem. Therefore, the original question of weak compactness of $\delta$ leads to the following operator equation.

$$
\text { Carn } \tilde{\delta_{x}}=0 \text { be solved in } K(A) \text { ? }
$$

Going one step further we can ask a sinilar question for the product $\delta_{1} \delta_{2}$ of two derivations $\delta_{1}, \delta_{2}$ on $A$ (which, in general, is no longer a derivation): when is $\delta_{1} \delta_{2}$ (weakly) compact?' 'This question should be related to the Dunford-Pettis property of a conmutative $C^{*}$-algebra which implies that $T_{1} T_{2}$ is a compact operator whenever $T_{1}, T_{2}$ are weakly compact on $A$. By similar arguments as above, it can be formulated in terms of operator equations as follows.

$$
\text { Can } \tilde{\delta}_{x_{1}} \tilde{\delta}_{s_{2}}=0 \text { be solved in } K(A) \text { ? }
$$

Questions of this kind are studied in [25] and [27].

\subsection{Completely positive operators.}

Recall that a linear mapping $T$ on a $C^{*}$-algebra $A$ is said to be completely bounded if the norms $\left\|T_{n}\right\|$ of the canonical exterisions $T_{n}$ of $T$ ro the matrix algebras $M_{n}(A)$ over $A$ are all bounded by some real number, and $T$ is completely positive if all $T_{n}$ are positive operators on $M_{n}(A)$. The prototypes of completely bounded operators are the elementary operators given concretely as mappings of the form

$$
S: x \mapsto \sum_{j=1}^{n} a_{j} x b_{j} \quad \text { with } x \in A, a_{1}, \ldots, a_{n}, b_{1}, \ldots, b_{n} \in M(A)
$$

where $M(A)$ denotes the multiplier algebra of $A$. This is justified by the representation theorem for conpletely bounded operators and the fact that certain completely bounded operators can be approximated 
by elementary operators, cf. [12]. A natural question in this context is: what does a completely positive elementary operator $S$ look like? Although this is involving incqualities, we immediately are led to an operator equation.

Denote by $M_{a, b}$ the (tur-sided) multiplication $x \mapsto$ axb. If $S=$ $\sum_{j=1}^{n} M_{a_{j}, b_{j}}$ is positive, it is hermitian-preserving from which

$$
\sum_{j=1}^{n} M_{a_{j,}, b_{j}}=\sum_{j=1}^{n} M_{b_{j}^{*}, a_{j}^{*}}
$$

follows. As a result we are to consider the following operator equation.

$$
\text { Which elements } x_{j}, y_{j} \in M(A) \text { solve } \sum_{j=1}^{+2} M_{x_{j}, y_{j}}=0 \text { ? }
$$

This question has emerged to be not only an example, but of fundamental significance for our approach, cf. [28].

\subsection{Centralizing mappings.}

Let $R$ be a ring. An additive mapping $F: R \rightarrow R$ is centralizing if, for every $x \in R$, we have $[x, F(x)]=x F(x)-F(x) x \in Z(R)$, the centcr of $R$. In many cases, the existence of certain centralizing mappings yields commutativity criteria for $R$. For example, if $R$ is a prime ring, then $R$ is commutative if there is a non-zero centralizing derivation on $R$ [38, Theorem 2], see also [30], or if there is a non-identical centralizing automorphism on $R$ [31, Theorem]. In the context of operator algebras, there are analogues of these results as follows.

Proposition 3. There is no non-zero centralizing derivation on a $C^{*}$-algebra.

This seems to be a folklore extension of Singer's classical result that there are no non-zero derivations on commutative $C^{*}$-algebras. In fact, if $\delta$ is a centralizing derivation on a $C^{*}$-algebra $A$, it easily follows that $\delta A \subseteq Z(A)$. Hence, the restriction $\delta$ of $\delta$ to $Z(A)$ vanishes so that $\delta^{2}=0$. The identity

$$
2 \delta(x) y \delta(x)=\delta^{2}(x y x)-x \delta^{2}(y x)-\delta^{2}(x y) x+x \delta^{2}(y) x \quad(x, y \in A)
$$

therefore yields $M_{\delta(x), \delta(x)}=0$ for all $x \in A$, whence $\delta=0$.

The case of automorphisms reguires some more work and was first studied by Miers. 
Proposition 4. [32, Theorem 5] Let $\alpha$ be a centralizing * automorphism on a von Neumann algebra $A$. There is a central projection $e \in A$ such that $\alpha(e)=e, \alpha_{\mid A e}=\mathrm{id}_{A e}$ and $A(1-e)$ is commutative.

Whether this result remains true for arbitrary (not necessarily *. preserving) automorphisms was answered only recently by Brešar; who also obtained a general structure theorem for centralizing mappings on von Neumann algebras as follows.

Proposition 5. [8, Theorem 2.1] Let $F$ be a centralizing additive mapping on a von Neumann algebra $A$. Then there exist an element $c \in Z(A)$ and an additive mapping $\zeta: A \rightarrow Z(A)$ such that $F=L_{c}+\zeta$.

Here and in the secuel, we will denote by $L_{a}$ the left multiplication $x \mapsto a x$ and by $R_{a t}$ the right mulliplication $x \mapsto x a$.

We will now reformulate both the assumption as well as the conclusion in terms of operator equations. This will enable us to obtain an extension of Brešar's result to arbitrary $C^{*}$-algebras in Section 4.

Observe at first that every centralizing additive ruapping $F$ on a $C^{*}$ algebra $A$ is in fact commuting, i.e. $[x, F(x)]=0$ for all $x \in A[9$, Proposition 3.1]. Replacing $x$ by $x+y$ therefore gives

$$
[x, F(y)]+[y, F(x)]=0 \quad(x, y \in A)
$$

or equivalently,

$$
\delta_{F(y)}-\delta_{y} F=0 \quad \text { for all } y \in A .
$$

Secondly, if $F=L_{c}+\zeta$ where $A$ is a $C^{*}$-subalgebra of a $C^{*}$-algcbra $B$ with centralizer $C_{B}(A), c \in C_{B}(A)$ and $\zeta: A \rightarrow C_{B}(A)$, then $[x, F(y)]=$ $[x, c y]+\{x, \zeta(y)]=[x, c y]$ for all $x, y \in A$. Hence

$$
[x, F(y)-c y]=0 \quad(x, y \in A)
$$

or equivalently,

$$
\delta_{F(y)-c y}=0 \quad \text { for all } y \in A .
$$

Conversely, if $c \in C_{B}(A)$ satisfes (3), then $\zeta=F-L_{c}$ defines an additive mapping from $A$ into $C_{B}(A)$. As a result we arrive at the following question.

(1.4) Suppose that, $F$ satisfies (2) for all $y \in A$. Is there an element $c \in C_{B}(A)$ for a 'suitable' $C^{*}$-algebra $B$ containing $A$ satisfying (3) for all $y \in A$ ?

Note that (3) precisely is a system of operator equations of the form (1) parametrized by all clements in $A$. 


\subsection{Generators of dynamical semigroups.}

Let $A$ be a unital $C^{*}$-algebra. A bounded herrnilian-preserving linear operator $L: A \rightarrow A$ with $L(1)=0$ is called completely dissipative if, for all $n \in \mathbb{N}$,

$$
L_{n}\left(x^{*} x\right) \geq x^{*} L_{n}(x)+L_{n}\left(x^{*}\right) x \quad\left(x \in M_{n}(A)\right) .
$$

These operators are the generators of norm-continuous one-parameter semigroups $\left(T_{t}\right)_{t \in \mathbb{R}_{+}}$of unital complctely positive operators $T_{t}$ or $A$, which describe the irreversible dynamics of open quantum systems, or; equivalcntly, serve as transition operators of non-commutative Markov processes. In many concrete situations, they are built from two prototypes: the completely posilive operators and the hermitian-preserving generalized inner derivations $\delta_{k, k^{*}}=R_{k}+L_{k^{*}}$. The converse question, when a given completely dissipative operator $L$ can be decornposed into

$$
L=\psi+\delta_{k, k}
$$

with $\psi$ completely positive from $A$ into some possibly larger $C^{*}$-algebra $B$ and $k \in B$ was first studied by Corini, Kossakowski and Sudarshan [18] and Lindblad $[21]$, and related to cohomological properties of $A$ in [22] and [11]. If $A \subseteq B(H)$, then a decomposition (4) of $L$ always exists with $\psi A \subseteq A^{\prime \prime}$ and $k \in A^{\prime \prime}$. In general, this decomposition will not be unique. The uniqueness problem can be reformulated in terms of an operator equation as follows. Suppose that

$$
L=\psi_{1}+\delta_{k_{\mathrm{t}}, k_{\mathrm{i}}^{*}}=\psi_{2}+\delta_{k_{2}, k_{2}^{*}}
$$

are two decompositions. Then, putting $a=k_{1}-k_{2}$, we have

$$
\delta_{a, a^{*}}+\psi_{1}-\psi_{2}=0
$$

Thus; we may ask

(1.5) Under which conditions does (5) imply that $\delta_{a, a^{*}}=0$ ?

A more general question would be which $a$ in $A^{\prime \prime}$ solve the equation (5).

\section{Devices}

All the above equations (1.1) through (1.5) can be subsumed under the general form (1). To motivate our tools for solving them, let us 
furthermore consider a special casc of (1.3). Let $A=B(H)$ and $b \in A$ be given.

(1.3') Which $a \in A$ solve $M_{r, t}=0$ ?

In our particular situation, the answer is quickly reached. If $M_{a, b}=0$, then $a x b \xi=0$ for all $x \in A$ and $\xi \in H$. If $b=0$, obviously all $a \in A$ are solutions. If $b \neq 0$, pick $\xi \in H$ with $b \xi \neq 0$ and note that $b \xi$ is cyclic for $A$, i.e. $A b \xi=H$, and thus $a=0$.

Clearly, this method only works in the presence of a Hilbert space on which $A$ acts 'transitively enough', $\Leftrightarrow$. if $A$ is irreducitse. The algebraic method presented now works withont underlying space.

It is convenient to rephrase (1.3) using the following concept. For every $C^{*}$-algebra $A$ we let $\mathcal{E l}(A)$ be the algebra of all elementary operators on $A$. We define a surjective algebra homomorphism

$$
\theta: M(A) \otimes M(\Lambda)^{\prime \prime \prime} \longrightarrow \mathcal{E}(A), \quad \theta(n \otimes b)=M_{a, b}
$$

where $M(A) \otimes M(A)^{o p}$ denotes the algebraic tensor product of $M(A)$ with its opposite algebrin. The problem now is to determine the kernel of $\theta$. The following was proved in [24, Part I, Corollary 4.4].

$$
\theta \text { is injective if and only if } A \text { is prime. }
$$

Since primitive $C^{*}$-algeloras are prime, it is tempting to use representalion theory in order to approach the general case from the special one. However, as it emerged, there may be problems in putting the 'local' information together to obtain in 'global' pichule. It, seens advantageous to vicw the prime $C^{*}$-algeloras as the bulding blocks, which results in regarding a $C^{* *}$-algebra as a semiprime algebra rather than an semisimple one. In fact; similar tedmiçues and resultis as those described below are available in the settimg of semiprime rings.

The ideal structure of a prime algebra is distinguished by the fact that every non-zero ideal is essenfiful, i.e. intersects cach other nonzero ideal non-trivially. This allows to "move around from one place to another" within the $C^{*}$-algebra without loss of information. For an arbitrary $C^{*}$-algebra $A$ we therefore denote by $\mathcal{I}_{c}$ and $\mathcal{I}_{c e}$ the collections of all essential and all closed essential ideals of $A$, respectively. Note that: these are directed downwards by inclusion, i.e. $I_{1}, I_{2} \in \mathcal{I}_{c}$ implies that $I_{1} \cap I_{2} \in \mathcal{I}_{c}$.

For every semiprine ring $R$, the multiplater ring $M(R)$ is defined by its universal property that $R$ is an essential idleal in $M(R)$ and there is 
a unique extension $\bar{\rho}$ of the inclusion $\rho: R \rightarrow M(R)$ which makes the following diagram commutative, whenever $R$ is an ideal in another ring $S$,

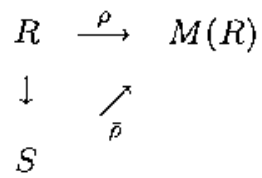

in other words, $M(R)$ is the (abstract) idealizer of $R$. Usually, $M(R)$ is constructed via double centralizers of $R$. Moreover, $\bar{\rho}$ is injective if and only if $R$ is essential in $S$. Now, if $I, J \in \mathcal{I}_{\text {cee }}$ and $J \subseteq I$, then $J$ will be an essential ideal in $M(I)$ whence, from the above, there is a unique injective *-homomorphism $\rho_{I J}: M(I) \rightarrow M(J)$ making the following diagram commutative

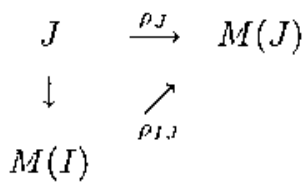

We may describe $\rho_{I J}$ as "restricting the double centralizers". By means of this, we obtain a directed system $\left\{M(I) ; \rho_{I J}, J \subseteq I\right\}$ of $C^{*}$-algebras and inclusions, and its algebraic direct limit alg $\lim M(I)$ along $\mathcal{I}_{c c}$ will be denoted by $Q_{b}(A)$ and called the bounded symmetric algebra of quotients of $A$. This is a pre- $C^{*}$-algebra with completion $Q_{b}(A)^{2}=\lim _{\longrightarrow} M(I)$ denoted henceforth by $M_{l o c}(A)$ and called the local multiplier algebra of A.

For each $I \in \mathcal{I}_{e}$ let $P(I)$ denote the Pcdersen ideal of $\bar{I}[37,5.6]$. Using the fact that $P(I)$ is ${ }^{*}$-invariant, belongs to $\mathcal{I}_{e}$, and that $P(I) P(J)=$ $P(I) \cap P(J)$ for all $I, J \in \mathcal{I}_{e}$ we defire $Q_{s}(A)=\underset{\rightarrow}{\operatorname{alg} \lim } M(P(I))$ along $\mathcal{I}_{c}$ and observe that this definition leads to the symmetric alyebra of quotients of $A$ as defined (slightly differently) in ring theory. It follows that $Q_{b}(A)$ embeds as a *-subalgebra into $Q_{s}(A)$ and is in fact the bounded part of $Q_{s}(A)$ [2, Theorem 1.3]. A stronger relation between $Q_{b}(A)$ and $Q_{s}(A)$ proved in $\left[3\right.$, Theorem 2] is that $Q_{s}(A)$ is the central localization of $Q_{b}(A)$.

Remarks. The construction of $M_{l o c}(A)$ was first performed by Pedersen [36] and Elliott [16] under the name of essential multipliers. They used it to study operator equations of the form

$$
\delta=\delta_{a}, \quad a \in M_{l o c}(A)
$$


ajd

$$
\alpha=M_{u, u^{*}}, \quad \psi \in M_{\text {loc }}(A) \text { ninitaly }
$$

that is, to obtain innerness of derivations $\delta$ and $*_{\text {-antomorphisms } \alpha \text { in }}$ $M_{\text {loc }}(A)$. In particular, Pedersen proved that (8) always has a solution if $A$ is separable $\left[36, P_{1}\right.$ poposition 2].

At about the same time, Khachenko introduced the symmetric ring of quotients for semiprine rings and used it in particular in Galois theory [19], [20]. This theme was further pursued by Passman [34], [35]; Montgomery [33]: and others. It is to be seen in a long tradition going back to the 30's in investigating general rings of ruoticnts, ef. [40]. The basic idea --- to enlarge n given 'dontan' by additional 'munbers" (='fractions': 'quotionts') in order to be able to solve more equations also serves as the motivetion for our appronch to operator cquations.

In the late 80 's, $M_{\text {loo }}(A)$ was rediscovered independently by Ara [2], [3] and the muthor [26], [29] which ther launched a joint research project on the structure and applications of local multipliers $[4],[5],\{6\}$; a comprehensive account of this is to be given in [7].

We will now compile some of the basic properties of $M_{\text {loc }}(A)$.

Proposition 6. Let A be a $C^{*}$-algebra with loch maltiplier algebra $M_{\text {loc }}(A)$.

(i) A is commutative if and only if $M_{\text {los }}(A)$ is commutative.

(ii) A is prime if artit only if $M_{\text {tor }}(A)$ has trivint conter.

(iii) For each $l \in I_{\text {ce }}$ and each unitization $B$ of $A$ we have

$$
M_{\text {loc }}(T)=M_{\text {loc }}(A)=M_{\text {loc }}(B)
$$

(iv) Let $\breve{A}$ be the primitive spectrurn of $A$. If $\check{A}$ is discrete, then $M_{l, c}(A)=M(A)$.

(v) If $A$ is an $A W^{*}$-algebra, then $M_{\text {loc }}(A)=A$.

Frow (7) and (ii) in the above proposition we sec that the kernel of $\theta$ is closely related to the center $Z=Z\left(M_{\text {toc }}(A)\right)$ of $M_{\text {loc }}(A)$. It is therefore important to analyse its structure. The following was proved in [5, Theorem 1 and Corollary 1] and can be viewed as a local version of the well-knowm Dams-Hofmanu theorem identifyng the center $Z(M(A))$ of $M(A)$ with the algebra $C(\beta \check{A})$ of all continuous complex-valued functions on the Store-Cech conpactification $\beta \ddot{A}$ of $\breve{A}$.

Proposition 7. For every $C^{*}$-algebra $A$, the center $Z$ of $M_{\text {Ioc }}(A)$ is an $A W^{*}$-algebra and can be identified with $C\left(\lim _{\longleftrightarrow} \beta \check{I}\right)$, where the inverse 
limit (in the category of compact spaces) is taken over all dense open subsets $\breve{I}$ of $\breve{A}$.

The key to this result is by observing that $Z=\overline{C_{b}}$, where $C_{b}=$ alg $\lim Z(M(I)), I \in \mathcal{I}_{c e}$, is the center of $Q_{b}(A)$ and called the bounded extended centroid of $A$. This one takes the role of the extended centroid $C=\underset{\rightarrow}{\operatorname{alg} \lim } Z(M(P(I))), I \in \mathcal{I}_{e}$, being of fundamental importance in ring theory. In analogy to the central closure $A C$ we define the bounded central closure ${ }^{c} A$ by ${ }^{c} A=\overline{A C_{b}}=\overline{A Z}$. The nicest $C^{*}$-algebras in this framework are those which are boundedly centrally closed, that is ${ }^{c} A=A$. They can be characterized as follows.

Proposition 8. $A$ is boundedly centrally closed if and only if $\dot{A}$ is extremally disconnected.

The fact that every von Neumann algebra is boundedly centrally closed (which follows in particular from Proposition $7(\mathrm{v})$ ) allows to incorporate the results on von Neumann algebras in our approach, and the fact that $M_{\text {toc }}(A)$ is boundedly centrally closed [5, Theorcm 2] yields an important, stability property.

It can be shown that every $C^{*}$-subalgebra $B$ of $M_{l o c}(A)$ containing both $A$ and $C_{b}$ has center $Z(B)$ equal to $Z[7]$, and hence may be regarded as a $Z$-bimodule in a natural way. Applying this to ${ }^{c} M(A)$, the bonnded central closure of $M(A)$, we obtain from (6) an induced homomorphism

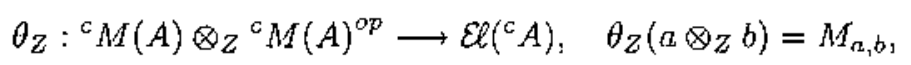

where the tensor product is taken in the category of birnodules. Using the fact that $A$ is boundedly centrally closed if and only if $M(A)$ is, we can now formulate the fundamental result yielding solutions to the opcrator equations listed in Section 2.

Theorem 9. [7] For every $C^{*}$-algebra $A$, we have that

$$
\text { ker } \theta=\left\{u \in M(A) \otimes M(A)^{o p} \mid u_{Z}=0\right\},
$$

where $u_{Z}$ is the canonical image of $u$ in ${ }^{c} M(A) \otimes_{Z}{ }^{c} M(A)^{o p}$. Therefore, if $A$ is boundedly centrally closed, then $\theta_{Z}$ is injective.

This result can be considerably strengthened using appropriate metric structures. Let $\mathcal{E}(A)$ be endowed with the $c b$-norm, i.e. $\|S\|_{c b}=$ 
$\sup \left\|S_{u}\right\|$ for all $S \in \mathcal{E}(A)$. Let ${ }^{c} M(A) \otimes{ }^{c} M(A)^{o p}$ be endowed with the central Haagerup tensor norm $\|\cdot\| z$ defined by

$$
\|u\|_{Z h}=\inf \left\{\left\|\sum_{j=1}^{n} a_{j} a_{j}^{*}\right\|^{1 / 2}\left\|\sum_{j=1}^{n} b_{j}^{*} b_{j}\right\|^{1 / 2} \mid u=\sum_{j=1}^{n} a_{j} \otimes Z b_{j}\right\}^{*}
$$

where the infimum is taken over all representations of $u$ in ${ }^{c} M(A) \otimes 2{ }^{c} M(A)^{o p}$. Then we have

Theorem 10. [7] For every $C^{*}$-algebra $A$;

$$
\theta_{Z}:\left({ }^{c} M(A) \otimes_{Z}{ }^{c} M(A)^{o p},\|\cdot\|_{Z H}\right) \rightarrow\left(\mathcal{E}\left({ }^{c} A\right),\|\cdot\|_{c b}\right)
$$

is an isometry.

Corollary 11. $\theta_{Z}$ is an isometry for every von Neumann algebra.

This last result was recently obtained in [10, Theorem 2.4], see also [39], for von Neumann algebras acting on separable Hilbert spaces using a number of non-trivial results on von Neumanin subfactors as well as direct integral theory.

\section{Solutions}

In this final section we will ontline answers to the questions raised in Section 2 exploiting the tools described in the previous section. As an immediate consequence of Theorem 9 we obtain the following answer to (1.3).

Theorem 12. Let $a=\left(a_{1}, \ldots, a_{n}\right), b=\left(b_{1}, \ldots, b_{n}\right) \in M(A)^{n}$ be such that $\left\{b_{1}, \ldots, b_{n}\right\}$ is $Z$-independert. If $\sum_{j=1}^{n} M_{a_{j}, b_{j}}=0$, then $a=0$.

Now the strategy to describe completely positive elenentary operators is as follows, cf. [7]. If $S=\sum_{j=1}^{\pi t} M_{t t_{j}, b_{j}}$ is completely positive, we may without loss of generality assume that both $\left\{a_{1}, \ldots, a_{n}\right\}$ and $\left\{b_{1}, \ldots, b_{n}\right\}$ are $Z$-independent. Then

$$
\sum_{j=1}^{\tau_{1}} M_{a_{j}, b_{j}}=\sum_{j=1}^{n} M_{b_{j}^{*}, a_{j}^{*}}
$$

*i.c., ${ }^{c} M(A) \otimes{ }_{h}{ }^{c} M(A)^{o p}$ interpits the operator space structure of ${ }^{c} M(A) \otimes_{h}{ }^{c} M(A)$. 
together with Theorem 12 implies the existence of a self-adjoint matrix $\Lambda=\left(\lambda_{k j}\right) \in M_{n}(Z)$ such that

$$
S=\sum_{k, j=1}^{n} \lambda_{k j} M_{b_{k}^{*}, b_{j}} .
$$

Since $Z$ is an $A W^{*}$-algebra by Proposition $7, \Lambda$ can be diagonalized by [14, Corollary 3.3], i.e. there is a unitary matrix $U \in M_{n}(Z)$ such that $U^{*} \Lambda U$ is diagonal with diagonal entries $\lambda_{1}, \ldots, \lambda_{n}$. Hence, by putting $\tilde{b}=b U^{*} \in{ }^{c} M(A)^{n}$ we can write $S$ as

$$
S=\sum_{j=1}^{n} \lambda_{j} M_{\tilde{b}_{j}^{+}, \bar{b}_{j}} .
$$

From the complete positivity of $S$ we then conclude that $\lambda_{j} \geq 0$ for all $1 \leq j \leq n$ and hence, letting $c_{j}=\lambda_{j}^{1 / 2} \bar{b}_{j}$, obtain the following answer to the question raised in 2.2 .

Theorem 13. [7] An elementary operator $S$ on a $C^{*}$-algebra $A$ is completely positive if and only if there are $c_{1}, \ldots, c_{n} \in{ }^{c} M(A)$ sach that $S=\sum_{j=1}^{n 2} M_{c_{j}^{*}, c_{j}}$.

For prime $C^{* *}$-algebras, this was obtained in [24, Part I, Theoren 4.10].

For simplicity, we stick to the prime case in answering the questions of Sections 2.1 and 2.4. If $A$ is a prime $C^{*}$-algebra, then, by Theorem 12, $R_{a}+L_{b}=0$ for some $a, b \in M(A)$ if and only if $a=-b \subset Z(M(A))=$ C1. Suppose that $\delta$ is a weakly compact derivation on $A$. If $\delta=0$, it clearly can be implemented by a compact element. If $\delta \neq 0$, then $\delta A \subseteq K(A)$ (Proposition I) implies that $K(A) \neq\{0\}$ and thus $A$ can be faithfully represented as an irreducible algebra on some Hilbert space $H$ such that $K(A)$ becomes $K(H)$. By the arguments used in 2.1, we see that $\delta=\delta_{a}$ for some $a \in B(H)$ and $\tilde{\delta}_{a}=\delta_{\tilde{a}}=0$ on the Calkin algebra $C(H)=B(H) / K(H)$. Since $C(H)$ is prime, $Z(C(H))=\mathbb{C l}$ wherefore $\vec{a}=\lambda 1$, equivalently, $a+\lambda 1 \in K(A)$. Consequently, we have the following.

Proposition 14. Let $\delta$ be a derivation on a prime $C^{*}$-algcbra $A$. Then $\delta$ is weakly compact if and only if $\delta=\delta_{a}$ for some $a \in K(A)$.

In fact, this result takes over verbatim to the case of a general $C^{*}$ algebra, which was first proved by Akemann and Wright [1, Theorem 
3.3] using representation thcory. As a result, a derivation $\delta$ is weakly compact if and only if the answer is "yes" in (1.1).

In a similar vein, $\delta_{1} \delta_{2}$ is weakly compact if and only if $\delta_{1}$ or $\delta_{2}$ is weakly compact, provided $A$ is prime. Hence, $\delta_{1} \delta_{2}$ is weakly compact if and only if at least one of the $x_{i}$ in (1.2) can be taken from $K(A)$. 'The formulation of the answer in the general case is somewhat more complicated, and we reler the reader for this (as well as for the norm compact (ase) to $[\mathbf{2 5}]$. Note that

$$
\delta_{x_{1}} \delta_{x_{2}}=M_{x_{1} x_{2}, 1}-M_{x_{1}, x_{2}}-M_{x_{2}, x_{1}}-M_{1, x_{2} x_{2}}
$$

and therefore (1.2) is closely related to (1.3) and a description of (weakly) compact elementary operators which was obtained in [24, Part II].

Specializing the above observation to the case $b=a^{*}$ we obtain that $\delta_{a, a^{*}}=0$ if and only if $a=-a^{*} \in i \mathbb{R} 1$ whenever $A$ is prime. Using a slight elaboration of this we obtain the answer to (1.5).

Theorem 15. Let $A$ be a unital $C^{*}$ algebra and $P$ a proper closed mime ideal of $A$. Let $L: A \rightarrow A$ be linear. Under the hypothesis $\psi A \subseteq P$, ench two decompositions of $L$ of the form $L=\psi+\delta_{k, k}$. with $\psi: A \rightarrow A$ completely positive and $k \in A$ only differ by an addition by $\delta_{\mathrm{c}, \mathrm{c} \mathrm{c}^{*},}, c \in P$.

Corollary 16. Let $A$ be a unital infinite dimensional prime $C^{*}$ algebra and $L: A \rightarrow A$. Then there is at most one decomposition $L=\psi+\delta_{k, k}$ with $k \in A$ and $\psi: A \rightarrow A$ completely positive and compact.

These results are proved in [17]. Corollary 16 was first observed by Davies ' 13 , Theorem 2] in the case $A=B(H)$.

We finally turn our attention to the structure of centralizing rrappings of $C^{*}$-algebras and the questions raised in Section 2.3. Unlike in the other examples, there seems to be no direct connection with equations involving elementary operators such as (1.3). The following leruma indeed is the key observation which enables us to solve equation (3).

Lemma 17. If $F$ is an arbitrary mapping on a ring $R$ such that $\delta_{F(y)}-\delta_{y} F$ maps $R$ into some ideal $J$ of $R$, then, for all $x, y, u, v \in R$, we have

$$
\left(M_{\delta_{F(y)}(x), \delta_{u}(v)}-M_{\delta_{y}(x) \cdot \delta_{F(u)}(v)}\right) R \subseteq J
$$

This result was obtained in $[8$, Lemma 2.2$]$ for commuting additive mappings and $J=\{0\}$. Although we are dealing here with $C^{*}$-algebras 
only, we give the proof in full generality as an illustration of the techniques and with a hope for future applications.

Proof: For all $y, z \in R$ we have

$$
\begin{aligned}
\delta_{y z} F & =\left(R_{y z}-L_{y z}\right) F=\left(R_{z} R_{y}-L_{y} L_{z}\right) F \\
& =\left(R_{z} \delta_{y}+L_{y} \delta_{z}\right) F=R_{z}\left(\delta_{y} F\right)+L_{y}\left(\delta_{z} F\right)
\end{aligned}
$$

and hence

$$
\delta_{F(y z)}-\delta_{y z} F=\delta_{F(y z)}-R_{z}\left(\delta_{y} F\right)-L_{y}\left(\delta_{z} F\right) .
$$

By assumption, it follows that

$$
\left(\delta_{F(y z)}-R_{z}\left(\delta_{y} F\right)-L_{y}\left(\delta_{z} F\right)\right) R \subseteq J .
$$

Observe that,

$$
\begin{aligned}
& \left(\delta_{F(y z)}-R_{z} \delta_{F(y)}-L_{y} \delta_{F(z)}\right)(x u)-L_{\mathrm{r}, y} \delta_{F(z)}(u)-R_{z u} \delta_{F(y)}(x) \\
& +R_{u z z} \delta_{F(y)}(x)+L_{y x} \delta_{F(z)}(u) \\
& =L_{x} \delta_{F(y z)}(u)+R_{u} \delta_{F(y z)}(x)-R_{z} L_{x} \delta_{F(y)}(u)-R_{z} R_{u} \delta_{F(y)}(x) \\
& -L_{y} L_{x} \delta_{F(z)}(u)-L_{y} R_{u} \delta_{F(z)}(x)-L_{x} L_{y} \delta_{F(z)}(u)-R_{u} R_{z} \delta_{F(y)}(x) \\
& +R_{z} R_{r_{t}} \delta_{F(y)}(x)+L_{y} L_{x} \delta_{F(z)}(u) \\
& =L_{x}\left(\delta_{F(y z)}-R_{z} \delta_{F(y)}-L_{y} \delta_{F(z)}\right)(u)+R_{u^{\prime}}\left(\delta_{F(y z)}-R_{z} \delta_{F(y)}-L_{y} \delta_{F(z)}\right)(x) \\
& =L_{x}\left(R_{z} \delta_{y} F+L_{y} \delta_{z} F-R_{z} \delta_{F(y)}-L_{y} \delta_{F(z)}\right)(u)+j_{1} \\
& +R_{u}\left(R_{z} \delta_{y} F+L_{y} \delta_{z} F-R_{z} \delta_{F(y)}-L_{y} \delta_{F(z)}\right)(x)+j_{2}
\end{aligned}
$$

(with $j_{1}, j_{2} \in J$ by (13))

$$
\begin{aligned}
=L_{x}\left(R _ { z } \left(\delta_{y} F-\right.\right. & \left.\left.\delta_{F(y)}\right)+L_{y}\left(\delta_{z} F-\delta_{F(z)}\right)\right)(u)+j_{1} \\
& +R_{u z}\left(R_{z}\left(\delta_{y} F-\delta_{F(y)}\right)+L_{y}\left(\delta_{z} F-\delta_{F(z)}\right)\right)(x)+j_{2} \in J
\end{aligned}
$$

since $\left(\delta_{y} F-\delta_{F(y)}\right) R \subseteq J$ by assumption.

By (13) again, the first summand on the left hand side is in $I$ too, from which we conclude that

$$
L_{x y-y x} \delta_{F(z)}(u)+R_{z u-u z} \delta_{F(y)}(x) \in J
$$

equivalently:

$$
\delta_{y}(x) \delta_{F(z)}(u)+\delta_{F(y)}(x) \delta_{u}(z) \in J
$$


for all $x, y, z, u \in R$.

Frorn

$$
\delta_{F(z)}(u)+\delta_{F(u)}(z) \in J \quad \text { for all } u, z \in R
$$

and (14) it follows that

$$
\delta_{y}(x) \delta_{F(u)}(z)-\delta_{F(y)}(x) \delta_{u}(z) \in J \quad \text { for all } x, y, z, u \in R .
$$

Replacing $z$ by $z v$ in (15) yields

$$
\begin{aligned}
& \delta_{y}(x) \delta_{F(u)}(z v)-\delta_{F(y)}(x) \delta_{u}(z v)= \\
= & \delta_{y}(x) z \delta_{F(u)}(v)+\delta_{y}(x) \delta_{P(u)}(z) v-\delta_{F(y)}(x) \delta_{u}(z) v-\delta_{F(y)}(x) z \delta_{u}(v) \in J
\end{aligned}
$$

which, together with (15), gives,

$$
\delta_{y}(x) z \delta_{F(u)}(v)-\delta_{F(y)}(x) z \delta_{u}(v) \in J
$$

for all $x, y, z, u, v \in R$. But (16) is nothing but the assertion.

As a consequence, every mpping satisfying (2) has the property that,

$$
M_{\delta_{F(y)}(x), \delta_{u}(v)}-M_{\delta_{y}(x), \delta_{F(u)}(u)}=0 \quad \text { for all } x, y, u, v \in A .
$$

An elaboration of the solution to (1.3), the cletails of which are given in [6], then yields a family $\left\{c_{x}^{y} \mid x, y \in A\right\}$ of elements in $C$ and a family $\left\{e_{x}^{y}\{x, y \in A\}\right.$ of projections in $C_{b}$, such that

$$
e_{y}^{y} \delta_{F(y)}(x)-c_{x}^{y} \delta_{y}(x)=0 \quad \text { for all } x, y \in A \text {. }
$$

It is then the self-injectivity of $C$ which allows to find $c \in C$ with $c e_{s}^{3 y}=$ $c_{x}^{y}$, which finally has the property that,

$$
\delta_{F(y)}(x)-c \delta_{y}(x)=0 \quad \text { for all } x, y \in A,
$$

that is, which solves (3). An ardditional argument is then needed to show that $c$ can be found in $C_{b}$, that is, we obtain a solution to (1.4) in ${ }^{c} A$.

We summarize this in the following statement.

Theorem 18. [6, Theorem 3.2] Let $F: A \rightarrow A$ be a centralizing additive mapping on a $C^{*}$-algebra $A$. Then there are $c \in Z$ and an additive mapping $\zeta: A \rightarrow Z$ such that $F=L_{c}+\zeta$.

Note that, by Proposition $6(v)$, this is an extension of Bresar's result (Proposition 5). Under a natural condition, both $c$ and $\zeta$ can be chosen uniquely. 


\section{Conclusion}

We hope that the results described above may give some evidence that the local multiplier algebra can serve as a 'universe', in which operator equations on $C^{*}$-algebras, at least those of the forin (1), can solved by a unified method.

\section{References}

1.. C. A. AKEMANN AND:S. WRIGHT, Compact and wcakly compact derivations of $C^{*}$-algebras, 'Pacific J. Math. 85 (1979), 253-259.

2. P. ARA, The extended centroid of 'Cं*algebras: Arch $:$ Math.' 54 (1990), 358-364.

3. P. ARA, On the symmetric algebra of quotients of a $C^{*}$-algebra, Glasgow Math. J, 32 (1990), 377-379.

4. P. ARA AND M. MATHEU, On ultraprime Banach algebras with non-zero socle, "Proc. R. "Ir. Acrd' 91 A (1991), "89-98.

5. P. ARA AND M. MATHEU, A local version of the Dauns-Hofrnann theorem, Math. Z. 208 (1991), 349-353.

6: P. ARA AND M. MATHUEU, An application of local multipliers to centralizing mappings on . $C^{*}$-algebras, Quart..J.. Math., Oxford, to appear.

7. P. ARA AND M. MATHIEU, Local nultipliers of $C^{*}$-algebras, in preparation.

8. M. BRES̆AR, Centralizing mappings on von Neumann algebras, Proc. Amer Math. Soc. 111 (1991), 501-510.

9. M. BREŠAR, Centralizing mappings and derivations in prime rings, J. Algebra, to appear.

10. A. Chatterjee and R. R. Smith, The central Haagerup tensor product and mạs between vơii Neumanin algebras, J. Funct. Añol, "to appear."

11. E. CHRISTENSEN AND' D.'E. EvANS, Cohomology of operator álgebras and quantum dyramical semigroups, J. London Math. Soc. 20 (1979), 358-368.

12. E. Christensen AND A. M. SINCLAIR, A Survey of completely bounded operators, Bull. London Math. Soc. 21 (1989), 417-448.

13. E. B. DAVIES Uniqueness of the standard form of the generator of a quantum dynamical semigroup: Rep. Math: Phys. 17 (1980); $249-255$. 
14. D. DECKart AND C. PEARCY, On matrices over the ring of continuous complex valued functions on a Stonian space, Proc. Amer. Math. Soc. 14 (1963), 322-328.

15. N. DunFord AND J. T. SCHWART', "Linear operalors", Part I, lriterscience, New York, 1958.

16. G. A. ElLIOTT, Automorphisms detemined by multipliers on ideats of a $C^{*}$-algebra, J. Funct. Anal. 23 (1976), 1-10.

17. T. Freiberger axd M. MATHeU, Uniqueness of a Lindblad decomposition, in Proc. Int. Workshop, on "Elementary Operators and Applicutions", Blaubeuren, Jume 1991, World Scientific, Singapore, 1992, pp. 179-187.

18. V. Gorini, A. Kossakowski nnd E. C. G. Sudarshan, Completely positive dymanical semigroups of $\mathrm{N}$-level systems, J. Math. Phys. 17 (1976). 821-825.

19. V. K. Kharchenko, Generalized identities with automorphisms, Algebra i Logika 14 (1975), 215-237, English transl. (1976), 132-148.

20. V. K. KHALCHENKo, Galois theory of semiprine rings, Algebra $i$ Logika 16 (1977), 313-363, English transl. (1978), 208-258.

21. G. LINDRIAJ), On the generators of quantum dynamical semigroups, Comminn. Muth. Phys. 48 (1976), 119-130.

22. G. LiNDBLAD, Dissipative operators and cohomology of operator algebras, Lett. Math. Phys. 1 (1976), $219 \cdot 224$.

23. M. MATIIEU, Generalising elcmentary operators, Semesterbericht Funktionalanalysis 14 (1988), 133-153.

24. M. MAs'HIEU, Elementary operators on prime $C^{*}$-algebras, 1 , Math. Ann. 284 (1989), 223-244; Il, Glasgow Math. J. 30 (1988), 275-284.

25. M. MATHISU, Properties of the product of two derivations of a C*-algebra, Canad. Math. Bull. 32 (1989), 490-497.

26. M. MATHeU, On $C^{*}$-ilgebras of quotients, Semesterbcricht Futhtionalanulysis 16 (1989), 107-120.

27. M. MNTHels, More properties of the product of two derivations of a $C^{*}$-algebra, Bull. Austral. Math. Soc. 42 (1990), 115-120.

28. M. Mathey, How to use primeness to describe properties of elementary operators, Proc. Sympos. Pure Math. 51, (Part II) (1990), $195-199$.

29. M. MATHEU, The synmetric algebra of equotients of an ultraprime Banach algebra, J. Austral. Math. Soc. (Series A) 50 (1991), 75-87.

30. M. Mathisu, Posner's second theorem deduced from the first, Proc. Amer. Math. Soc. 114 (1992), 601-602. 
31. J. H. MAYNE, Centralizing automorphisms of prime rings, Canarl. Math. Bull. 19 (1976), 113-115.

32. C. R. Miers, Centralizing mappings of operator algebras, J. Algebra 59 (1979), 56-64.

33. S. MONTGOMERY, "Fixed rings of finite automorphism groups of associative rings," Lect. Notes Math. 818, Springer-Verlag, Berlin Heidelberg New York, 1980.

34. D. S. PASSMAN, "Group rings, crossed products and Galois theory," CBMS Series 64, Amer. Math. Soc, Providence, RI, 1986.

35. D. S. PASSMAN, Computing the symmetric ring of quotients, $J$. Algebra 105 (1987), 207-235.

36. G. K. PeDersen, Approximating derivations on ideals of $C^{*}$ algebras, Invent. Math. 45 (1978), 299-305.

37. G. K. Pedersen, "C $C^{*}$-algebras and their automorphism groups;" Academic Press, London, 1979.

38. E. C. Posner, Derivations in prime rings, Proc. Amer. Math. Soc. 8 (1957), 1093-1100.

39. R. R. Sмгтн, Elementary operators and the Haagerup tensor product, in Proc. Int. Workshop, on "Elementary Operators and Applications", Blaubeuren, June 1991, World Scientific, Singapore, 1992, pp. 233-241.

40. B. Ste.sström, "Rings of quotients," Grundlehren d. Math. Wiss. 217, Springer-Verlag, Berlin Heidelberg New York, 1975.

41. K. YLINEN, Weakly completely continuous elements of $C^{*}$-algebras; Proc. Amer. Math. Soc. 52 (1975), 323-326.

Mathematisches Institut der Universität Tübingen

Aul der Morgenstelle 10

D-7400 'Tübingen

GERMANY

Rebut el 9 de Gener de 1992 